\title{
Chlorella virus pyrimidine dimer glycosylase and Escherichia coli endonucleases IV and V have incision activity on 2,2,4-triamino- $5(2 H)$-oxazolone
}

\author{
Katsuhito Kino ${ }^{1 *}$, Masayo Suzuki ${ }^{1}$, Masayuki Morikawa' ${ }^{1}$, Takanobu Kobayashi', Shigenori Iwai ${ }^{2}$ and Hiroshi Miyazawa ${ }^{1}$
}

\begin{abstract}
Introduction: 2,2,4-Triamino-5(2H)-oxazolone $(\mathrm{Oz})$ in a DNA strand is an oxidation product of guanine and 8-oxo-7, 8-dihydroguanine, and such a lesion can cause G-to-C transversions. Previously, Fpg/Nei and Nth were shown to have incision activity on $\mathrm{Oz}$.

Findings: We investigated the activities of chlorella virus pyrimidine dimer glycosylase (cvPDG) and Escherichia coli endonucleases IV (Nfo) and V (Nfi) on Oz. Although the three enzymes have different repair mechanisms from Fpg/Nei and $\mathrm{Nth}$, they still had incision activity on $\mathrm{Oz}$.

Conclusions: Given the incision activities of CvPDG, Nfo and Nfi on Oz in addition to Fpg/Nei and Nth, Oz is DNA damage that can be repaired by diverse enzymes.

Keywords: Guanine oxidation, Oxazolone, Chlorella virus pyrimidine dimer glycosylase, Escherichia coli endonucleases IV, Escherichia coli endonucleases V
\end{abstract}

\section{Introduction}

Endogenous and exogenous oxidative stress causes DNA damage, and several enzymes repair this damage $[1,2]$. Among the four bases, guanine is most susceptible to oxidative damage. Although 8-oxo-7,8dihydroguanine (8oxoG) (Fig. 1a) is known to be a guanine oxidation product and a typical oxidation marker, 8oxoG has lower oxidation potential than guanine and is more readily oxidized. Thus, 8oxoG can be further oxidized, and oxidation products other than 8oxoG would be expected to have biological effects. 2,2,4-Triamino-5(2H)-oxazolone $(\mathrm{Oz})$ is produced from guanine and $80 x 0 \mathrm{G}$ by several oxidants: one-electron transfer [3]; superoxide radical [4]; singlet oxygen [5]; hydroxyl radical [6]; $\gamma$-radiation [3]; peroxynitrite [7]; and iodine [8]. Two to six molecules of $\mathrm{Oz}$ per $10^{7}$ guanines have been detected in liver

\footnotetext{
* Correspondence: kkino@kph.bunri-u.ac.jp

'Kagawa School of Pharmaceutical Sciences, Tokushima Bunri University,

1314-1 Shido, Sanuki, Kagawa 769-2193, Japan

Full list of author information is available at the end of the article
}

DNA [9]. Although Escherichia coli DNA polymerase inserted adenine opposite $\mathrm{Oz}$ and $\mathrm{Oz}$ caused G-to-T transversions in $E$. coli cells $[10,11]$, we recently reported that eukaryotic DNA polymerases $\alpha, \beta, \delta$, and $\varepsilon$ almost exclusively inserted guanine opposite $\mathrm{Oz}$ $[12,13]$ and incorporation of adenine was dependent on families of DNA polymerases [12]. Importantly, cytosine can be incorporated opposite 8oxoG, but not opposite $\mathrm{Oz}$. Therefore, $\mathrm{Oz}$ is a pre-mutagenic lesion that can cause G-to-C or G-to-T transversions in eukaryotes, and so repair of $\mathrm{Oz}$ is required to prevent point mutations.

Previously, E. coli Fpg and Nth enzymes were shown to excise $\mathrm{Oz}$ from dsDNA oligomers with similar efficiencies regardless of the type of base in the opposite strand $[10,14]$. Recently, we found that human NEIL1 (hNEIL1) and NTH1 (hNTH1) can excise Oz [15]. Herein, we report the incision efficiency of chlorella virus pyrimidine dimer glycosylase (cvPDG) and E. coli endonucleases IV (Nfo) and V (Nfi) on Oz. 
<smiles>Nc1nc2c(=O)[nH]c(=O)[nH]c2n1C1CC(O)C(O)C1</smiles>

80xoG

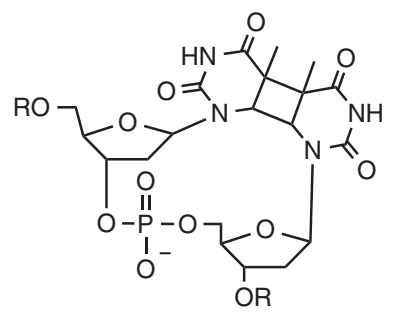

PD<smiles>N=C(N)N=C(NC1CC(O)C(CO)O1)C(=O)O</smiles>

$\mathrm{Oz}$<smiles>O=c1[nH]cnc2c1ncn2C1OC(O)CC1O</smiles>

H<smiles>N=C(N)NC1C(=O)NC(=O)N1C1CC(O)C2(O)CC1O2</smiles>

Gh

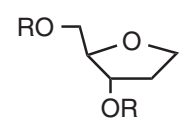

THF
(B)

$5^{\prime}-*$ CTCGTCAGCA TCPDCATCAT ACAGTCAGTG 3'- GAGCAGTCGT AGAAATAGTA TGTCAGTCAC

(C)

$51-*$ CTCATCAACA TCTTXAATTC ACAATCAATA

3 ' - GAgTAGTTGT AGAAȲTTAAG TGTTAgTtAT
(D)

\begin{tabular}{ccc}
\hline Name & $\mathrm{X}$ & $\mathrm{Y}$ \\
\hline 8oxoG:C & 8oxoG & $\mathrm{C}$ \\
Oz:C & $\mathrm{Oz}$ & $\mathrm{C}$ \\
Gh:C & $\mathrm{Gh}$ & $\mathrm{C}$ \\
$\mathrm{H}: \mathrm{T}$ & $\mathrm{H}$ & $\mathrm{T}$ \\
THF:A & THF & $\mathrm{A}$
\end{tabular}

Fig. 1 Structures of DNA damages and sequences containing DNA damages. a Structures of guanine oxidation products, cyclobutane thymine dimer (PD), hypoxanthine (H) and tetrahydrofuran (THF). $\mathbf{b}$ The sequence of the DNA duplex containing PD. The top strand contained PD and was labeled with ${ }^{32} \mathrm{P}$ at the $5^{\prime}$ end (indicated by ${ }^{*}$ ). $\mathbf{c}$ The sequences of the DNA substrates. The top strand contained a lesion at position $\mathrm{X}$ and was labeled with ${ }^{32} \mathrm{P}$ at the $5^{\prime}$ end (indicated by $\left.{ }^{*}\right)$. $\mathbf{d} \mathrm{X}$ and $\mathrm{Y}$ in the nucleotides represent each lesion

\section{Materials and methods Materials}

Oligonucleotides containing a single Oz, guanidinohydantoin (Gh) and cyclobutane thymine dimer (PD) were prepared as described previously [10, 16-18]. The oligonucleotides containing a single 8oxoG, hypoxanthine (H) and tetrahydrofuran (THF) were purchased from Nihon BioService. Nfo and Nfi were purchased from New England Biolabs. cvPDG was purchased from Trevigen.

\section{PAGE analysis of nicking reactions with enzymes}

The oligonucleotide (30mer) containing $\mathrm{Oz}, \mathrm{Gh}, \mathrm{PD}$, hypoxanthine, 8oxoG and THF was $5^{\prime}$ end-labeled by treatment with $\mathrm{T} 4$ polynucleotide kinase and $\left[\gamma^{3}{ }^{32} \mathrm{P}\right] \mathrm{ATP}$ and purified. The sequences of the oligomers used are shown in Fig. $1 \mathrm{~b}-\mathrm{d}$. The nicking reactions $(5 \mu \mathrm{l})$ were performed in mixtures containing the following components: (for Nfo) $50 \mathrm{mM}$ Tris- $\mathrm{HCl}, \mathrm{pH} 7.9,100 \mathrm{mM}$ $\mathrm{NaCl}, 10 \mathrm{mM} \mathrm{MgCl} 2,1 \mathrm{mM}$ DTT, and $0.5 \mu \mathrm{g}$ BSA, (for Nfi) $20 \mathrm{mM}$ Tris-acetate, $\mathrm{pH}$ 7.9, $50 \mathrm{mM}$ potassium acetate, $10 \mathrm{mM}$ magnesium acetate, $1 \mathrm{mM}$ DTT, and $0.5 \mu \mathrm{g}$ BSA, (for cvPDG) $25 \mathrm{mM}$ sodium phosphate, $\mathrm{pH}$ 6.8, $100 \mathrm{mM} \mathrm{NaCl}, 1 \mathrm{mM}$ EDTA, $1 \mathrm{mM}$ DTT, and $1 \mu \mathrm{g}$ BSA. The ${ }^{32}$ P-labeled DNA (100 fmol), complementary oligomer $(200 \mathrm{fmol})$ and each enzyme were incubated at $30{ }^{\circ} \mathrm{C}$ for $1 \mathrm{~h}$. Reactions with enzymes were stopped by adding an equal volume of dye solution containing EDTA, heated at $70{ }^{\circ} \mathrm{C}$ for $5 \mathrm{~min}$, and subjected to $16 \%$ denaturing PAGE. Radioactivity was quantified using the BAS2500 bioimaging analyzer (Fujifilm).

\section{Results and discussion}

The incision of oligonucleotides containing $\mathrm{Oz}$ by pyrimidine dimer glycosylase

cvPDG cleaves glycosidic bonds of the $5^{\prime}$-pyrimidine of a cyclobutane pyrimidine dimer, followed by cleavage of phosphodiester bonds (Fig. 2a) [19, 20]. Moreover, 8oxoG is not a substrate of cvPDG, but formamidopyrimidines 


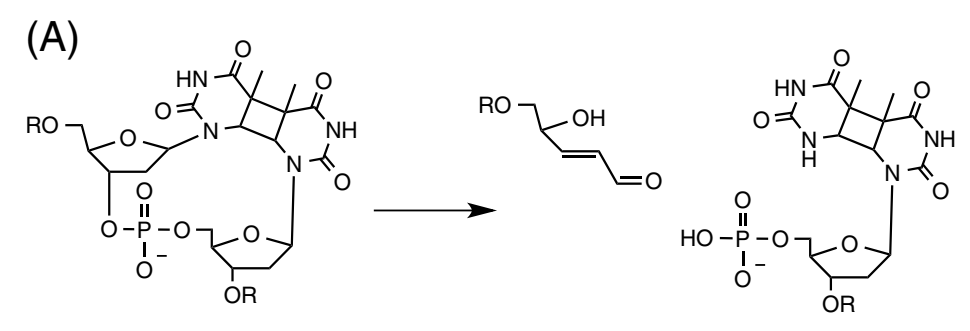

PD

(B)

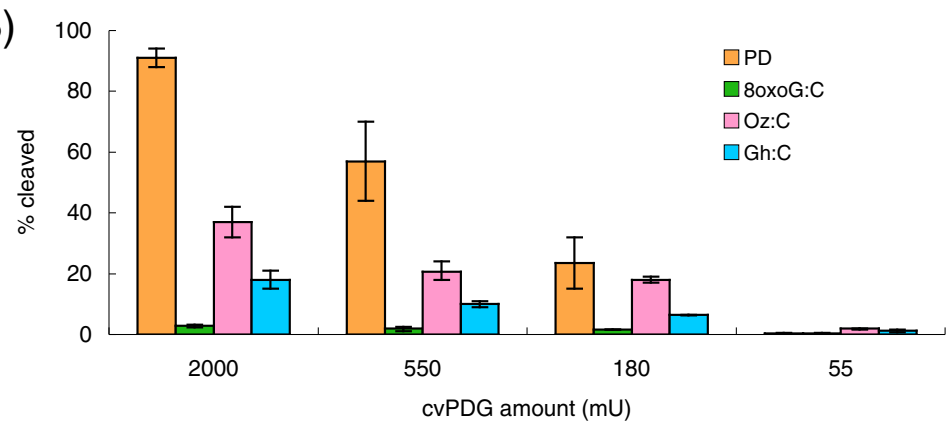

Fig. 2 Incision activities of chlorella virus pyrimidine dimer glycosylase (cVPDG). a The scheme of the cleaved oligonucleotides containing PD (Fig. 1) using CVPDG $[19,20]$. b The percentage of the cleaved oligonucleotides in the ${ }^{32}$ P-labeled DNA substrate with varied amounts of cvPDG was plotted as a graph. The mean values and standard errors were calculated from at least two independent experiments

[21]. Thus, we investigated the incision activities of cvPDG on Oz-containing duplex DNA, which were compared with those on $\mathrm{PD}$ as the positive control lesion (Fig. 2b). Figure $2 \mathrm{~b}$ shows that $\mathrm{Oz}$ is able to be cleaved by cvPDG, but the observed activities on $\mathrm{Oz}$ are lower than those on PD. Thus, in addition to formamidopyrimidines [21], Oz is also a substrate of cvPDG.

Gh is an oxidation product of 8oxoG, and DNA polymerases incorporate adenine and guanine opposite $\mathrm{Gh}$ [16]. Since the behavior of Gh in DNA replication somewhat resembles that of $\mathrm{Oz}$, we compared the activity on $\mathrm{Gh}$ with $\mathrm{Oz}$. Figure $2 \mathrm{~b}$ shows that cvPDG incises $\mathrm{Oz}$ more efficiently than $\mathrm{Gh}$, and much more than 8oxoG. It was previously reported that the order of piperidine reactivity is $\mathrm{Oz}>\mathrm{Gh}>$ 8oxoG, and the order of the $N$-glycosidic bond strength is $\mathrm{Oz}<\mathrm{Gh}<$ 8oxoG [15]. When DNA glycosylases do not recognize a specific lesion, cleavage of glycosidic bonds by DNA glycosylases is related to the $N$-glycosidic bond strength [15]. Thus, moderate repair efficiency of $\mathrm{Oz}$ by cvPDG seems to be due to weak $N$-glycosidic bond strength at $\mathrm{Oz}$ rather than accurate recognition of $\mathrm{Oz}$ by cvPDG.

\section{The incision of oligonucleotides containing $\mathrm{Oz}$ by endonuclease IV (Nfo)}

Nfo is an apurinic/apyrimidinic endonuclease, and it hydrolyzes the phosphodiester bond $5^{\prime}$ to an abasic site
(Fig. 3a) [19, 22]. The catalytic mechanism of Nfo is quite different from that of Nei, Nth, hNEIL1 or hNTH1. We investigated the incision activities of Nfo on the Oz-containing duplex DNA and compared them with those on THF (Fig. 1a) as a stable mimic of the abasic site. The results in Fig. 3b indicate that the observed activity of $\mathrm{Nfo}$ on $\mathrm{Oz}$ is one-third to one-fourth of that on THF. Thus, Nfo can moderately repair Oz.

In Fig. 3b, Nfo incised Oz more efficiently than Gh. Since $\mathrm{Gh}$ has a five-membered ring structure, Gh is less similar to the abasic site than Oz (Fig. 1a). Thus, it seems that Nfo repairs Oz more efficiently than Gh due to its similarity to the abasic site. Taken together, Nfo appears to be better suited for being a backup repair enzyme for $\mathrm{Oz}$, than for $\mathrm{Gh}$.

\section{The incision of oligonucleotides containing $\mathrm{Oz}$ by endonuclease $\mathbf{V}$ (Nfi)}

Nfi is a deoxyinosine 3' endonuclease. Nfi cleaves at the second phosphodiester bond $3^{\prime}$ to the hypoxanthine residue (Fig. 3c) [19, 23], and the catalytic mechanism of Nfi is quite different from that of Nei, Nth, hNEIL1 or hNTH1. It is possible that Nfi recognizes a wide variety of substrates [23]. Therefore, we investigated the incision activity of Nfi on the Oz-containing duplex DNA, which were compared with that on hypoxanthine (Fig. 1a) as the positive control lesion. In addition, the incision activity of Nfi at the $\mathrm{Oz}$ lesion was compared with that at 
(A)

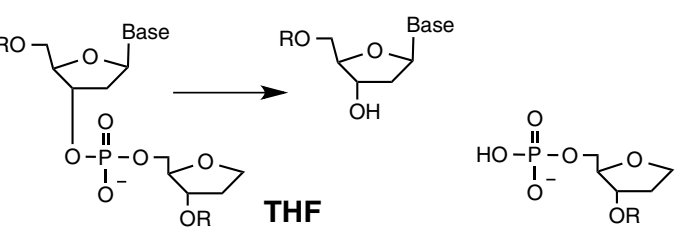

(B)
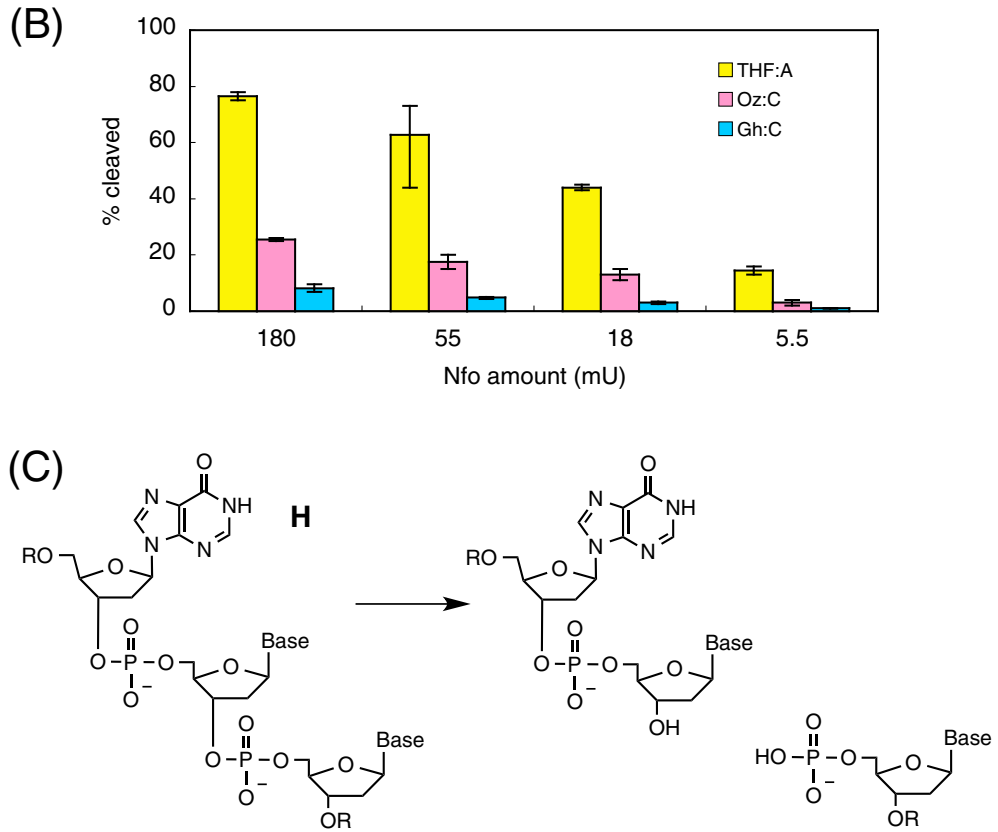

(D)

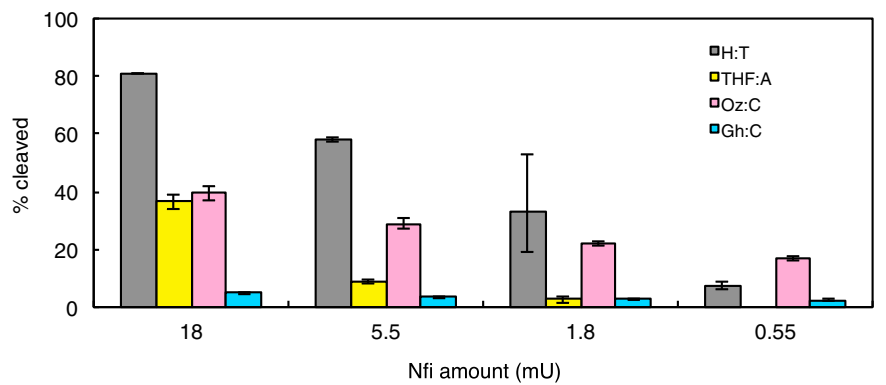

Fig. 3 Incision activities of E. coli endonuclease IV (Nfo) and endonuclease $V(\mathrm{Nfi})$. a,c The scheme of the cleaved oligonucleotides using Nfo $[19,21](\mathbf{a})$ and $\mathrm{Nfi}[19,22]$ (c). b,d The percentage of the cleaved oligonucleotides in the ${ }^{32} \mathrm{P}$-labeled DNA substrate with varied amounts of $\mathrm{Nfo}$ (b) and Nfi (d) was plotted as a graph. The mean values and standard errors were calculated from at least two independent experiments

THF. The results in Fig. 3d indicate that the observed activity on $\mathrm{Oz}$ is lower than that on hypoxanthine at high concentrations of Nfi, and is higher than that on THF. Since THF has no base moiety, Nfi does not readily recognize $\mathrm{THF}$, and these data indicate that $\mathrm{Oz}$ has some recognition sites for Nfi. Since Nfi recognizes oxanine (Fig. 4a) in DNA [23] and $\mathrm{Oz}$ is possible to have the open- or closed-ring structure (Fig. 4a), the closed-ring structure of $\mathrm{Oz}$ similar to oxanine (Fig. 4a) is thought to be able to react with Nfi.

It was shown that Nfi was able to recognize $\mathrm{Oz}$ much more efficiently than Gh (Fig. 3d). Previously, Nfi was shown to recognize uracil but not thymine in DNA, suggesting that the $5^{\prime}$-methyl group is critical for recognition by Nfi (Fig. 4b) [23]. Gh has a moiety protruding from the ring as does thymine (Fig. 4b), thus Nfi may have low incision activity against oligonucleotides containing Gh. Nfi is therefore better suited for being a backup repair enzyme for $\mathrm{Oz}$, than for $\mathrm{Gh}$.

\section{Implications and conclusion}

We described our analysis of incision reactivities on $\mathrm{Oz}$ with various repair enzymes. Human 8-oxoguanine DNA N-glycosylase 1 and human apurinic/apyrimidinic 


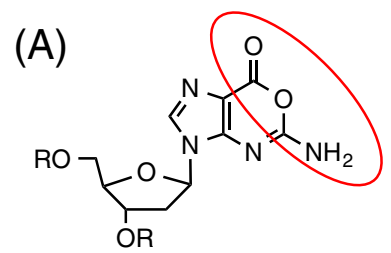

Oxanine

(B)

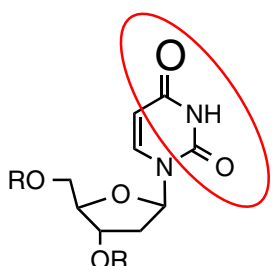

Uracil

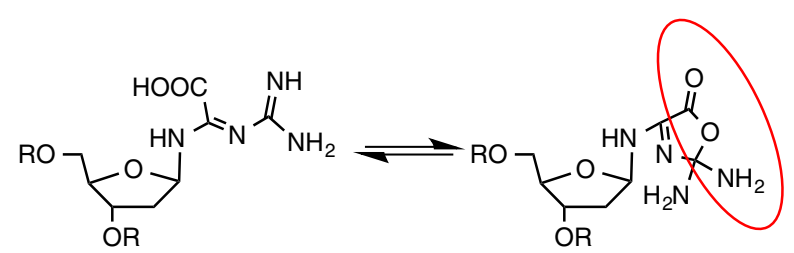

$\mathrm{Oz}$ (closed-ring)

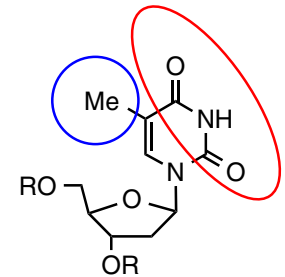

Thymine

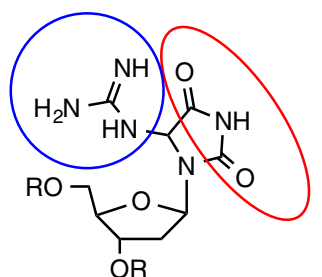

Gh

Fig. 4 Structures of DNA lesions and possible recognition site of Nfi. a Structures of oxanine and the possible open- or closed-ring structure of $\mathrm{Oz}$. Red circle indicates the common moiety that Nfi may recognize. b Structures of uracil, thymine and Gh. Red circle indicates the common moiety that Nfi may recognize. Blue circle indicates the moiety protruding from the six-or five-membered ring, and Nfi may disfavor these moieties

endonuclease 1 have no activity on $\mathrm{Oz}[15,24]$, thus these enzymes are not repair enzymes for Oz. In contrast, hNEIL1 and hNTH1 enzymes have high reactivities on $\mathrm{Oz}$, even though these enzymes erroneously incise at $\mathrm{Oz}$ sites of Oz:G and Oz:A [15]. The results in this paper reveal that Nfo, Nfi and cvPDG also have moderate activities on $\mathrm{Oz}$ compared with each positive control lesion. Nonetheless, these three enzymes incised $\mathrm{Oz}$ more efficiently than $\mathrm{Gh}$, thus they may serve as a backup for repair of $\mathrm{Oz}$.

\section{Abbreviations}

cvPDG: Chlorella virus pyrimidine dimer glycosylase; Nfo: E. coli endonuclease IV; Nfi: E. coli endonuclease V; hNEIL1: human NEIL1; hNTH1: human NTH1;

Oz: 2,2,4-Triamino-5(2H)-oxazolone; 8oxoG: 8-oxo-7,8-dihydroguanine; Gh: guanidinohydantoin; PD: cyclobutane thymine dimmer; $\mathrm{H}$ : hypoxanthine; THF: tetrahydrofuran.

\section{Competing interests}

The authors declare that they have no competing interests.

\section{Authors' contributions}

KK: conception and design of the study. KK: PAGE analysis. KK, MS, MM, SI: DNA synthesis. KK, MS, TK, SI, HM: drafting of the manuscript. KK, HM: critical revision of the manuscript for important intellectual content and supervision. All authors read and approved the final manuscript.

\section{Acknowledgements}

This work was supported by a research grant from the Japan Prize Foundation.

\section{Author details}

'Kagawa School of Pharmaceutical Sciences, Tokushima Bunri University, 1314-1 Shido, Sanuki, Kagawa 769-2193, Japan. 'Division of Chemistry, Graduate School of Engineering Science, Osaka University, Osaka, Japan.
Received: 27 July 2015 Accepted: 17 September 2015

Published online: 01 November 2015

\section{References}

1. Bjelland S, Seeberg E. Mutagenicity, toxicity and repair of DNA base damage induced by oxidation. Mutat Res. 2003;531:37-80.

2. Neeley WL, Essigmann JM. Mechanisms of formation, genotoxicity, and mutation of guanine oxidation products. Chem Res Toxicol. 2006;19:491-505.

3. Cadet J, Berger M, Buchko GW, Joshi PC, Raoul S, Ravanat J-L. 2,2-Diamino4-[(3,5-di-O-acetyl-2-deoxy- $\beta$-D-erythro-pentofuranosyl)amino]-5-(2H)oxazolone: a novel and predominant radical oxidation product of $3^{\prime}, 5^{\prime}-$ di-Oacetyl-2'-deoxyguanosine. J Am Chem Soc. 1994;116:7403-4.

4. Misiaszek R, Crean C, Joffe A, Geacintov NE, Shafirovich V. Oxidative DNA damage associated with combination of guanine and superoxide radicals and repair mechanisms via radical trapping. J Biol Chem. 2004;279:32106-15.

5. Ravanat J-L, Cadet J, Araki K, Toma HE, Medeiros MHG, Mascio PD. Supramolecular cationic tetraruthenated porphyrin and light-induced decomposition of 2'-deoxyguanosine predominantly via a singlet oxygenmediated mechanism. Photochem Photobiol. 1998;68:698-702.

6. Adam W, Berger M, Cadet J, Dall'Acqua F, Epe B, Frank S, et al. Photochemistry and photobiology of furocoumarin hydroperoxides derived from imperatorin: novel intercalating photo-Fenton reagents for oxidative DNA modification by hydroxyl radicals. Photochem Photobiol. 1996;63:768-78.

7. Douki T, Cadet J. Peroxynitrite mediated oxidation of purine bases of nucleosides and isolated DNA. Free Rad Res. 1996;24:369-80.

8. Kino K, Morikawa M, Kobayashi T, Kobayashi T, Komori R, Sei Y, et al. The oxidation of 8-oxo-7,8-dihydroguanine by iodine. Bioorg Med Chem Lett. 2010;20:3818-20.

9. Matter B, Malejka-Giganti D, Csallany AS, Tretyakova N. Quantitative analysis of the oxidative DNA lesion, 2,2-diamino-4-(2-deoxy- $\beta$-D-erythropentofuranosyl)amino]-5(2H)-oxazolone (oxazolone), in vitro and in vivo by isotope dilution-capillary HPLC-ESI-MS/MS. Nucleic Acids Res. 2006;34:5449-60.

10. Duarte V, Gasparutto D, Jaquinod M, Cadet J. In vitro DNA synthesis opposite oxazolone and repair of this DNA damage using modified oligonucleotides. Nucleic Acids Res. 2000;28:1555-63.

11. Henderson PT, Delaney JC, Gu F, Tannenbaum SR, Essigmann JM. Oxidation of 7,8-dihydro-8-oxoguanine affords lesions that are potent sources of replication errors in vivo. Biochemistry. 2002;41:914-21. 
12. Kino K, Sugasawa K, Mizuno T, Bando T, Sugiyama H, Akita M, et al. Eukaryotic DNA polymerase $\alpha, \beta$ and $\varepsilon$ incorporate guanine opposite 2,2,4-triamino-5(2H)-oxazolone. ChemBioChem. 2009;10:2613-6.

13. Suzuki M, Kino K, Kawada T, Morikawa M, Kobayashi T, Miyazawa H. Analysis of nucleotide insertion opposite 2,2,4-triamino-5(2H)-oxazolone by eukaryotic B- and Y-family DNA polymerases. Chem Res Toxicol. 2015;28:1307-16.

14. Tretyakova NY, Wishnok JS, Tannenbaum SR. Peroxynitrite-induced secondary oxidative lesions at guanine nucleobases: chemical stability and recognition by the Fpg DNA repair enzyme. Chem Res Toxicol. 2000;13:658-64.

15. Kino K, Takao M, Miyazawa $H$, Hanaoka F. A DNA oligomer containing 2,2,4-triamino-5(2H)-oxazolone is incised by human NEIL1 and NTH1. Mutat Res. 2012;734:73-7.

16. Kornyushyna O, Berges AM, Muller JG, Burrows CJ. In vitro nucleotide misinsertion opposite the oxidized guanosine lesions spiroiminodihydantoin and guanidinohydantoin and DNA synthesis past the lesions using Escherichia coli DNA polymerase I (Klenow fragment). Biochemistry. 2002:41:15304-14.

17. Ikeda H, Saito I. 8-Methoxydeoxyguanosine as an effective precursor of 2-aminoimidazolone, a major guanine oxidation product in one-electron oxidation of DNA. J Am Chem Soc. 1999;121:10836-7.

18. Murata T, Iwai S, Ohtsuka E. Synthesis and characterization of a substrate for T4 endonuclease $V$ containing a phosphorodithioate linkage at the thymine dimer site. Nucleic Acids Res. 1990;18:7279-86.

19. Friedberg EC, Walker GC, Siede W, Wood RD, Schultz RA, Ellenberger T. DNA repair and mutagenesis. 2nd ed. Washington DC: ASM; 2006.

20. Garvish JF, Lloyd RS. Active-site determination of a pyrimidine dimer glycosylase. J Mol Biol. 2000;295:479-88.

21. Jaruga P, Jabil R, McCullough AK, Rodriguez H, Dizdaroglu M, Lloyd RS. Chlorella virus pyrimidine dimer glycosylase excises ultraviolet radiationand hydroxy radical-induced products 4,6-diamino-5-formamidopyrimidine and 2,6-diamino-4-hydroxy-5-formamidopyrimidine from DNA. Photochem Photobiol. 2002;75:85-91.

22. Ide H, Tedzuka K, Shimzu H, Kimura Y, Purmal AA, Wallace SS, et al. a-Deoxyadenosine, a major anoxic radiolysis product of adenine in DNA, is a substrate for Escherichia coli endonuclease IV. Biochemistry. 1994:33:7842-7.

23. Feng $H$, Dong $L$, Klutz AM, Aghaebrahim N, Cao W. Defining amino acid residues involved in DNA-protein interactions and revelation of $3^{\prime}$-exonuclease activity in endonuclease V. Biochemistry. 2005:44:11486-95.

24. Kino K, Sugasawa K, Sugiyama H, Miyazawa H, Hanaoka F. The base excision repair reaction of oxazolone with hOGG1. Photomed Photobiol. 2004;26:41-2

\section{Submit your next manuscript to BioMed Central and take full advantage of:}

- Convenient online submission

- Thorough peer review

- No space constraints or color figure charges

- Immediate publication on acceptance

- Inclusion in PubMed, CAS, Scopus and Google Scholar

- Research which is freely available for redistribution

Submit your manuscript at www.biomedcentral.com/submit 